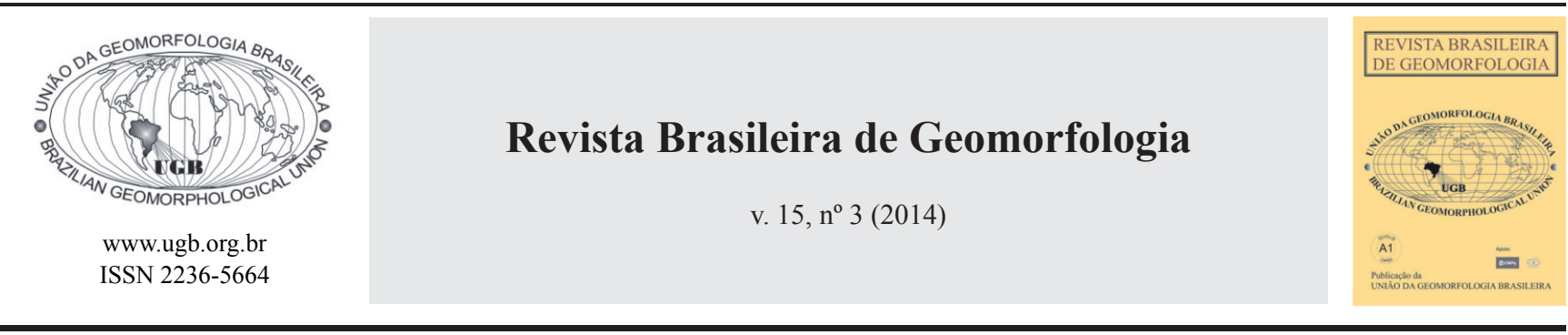

\title{
RELAÇÃO ENTRE A GEOMETRIA HIDRÁULICA E A CURVA DE PERMANÊNCIA EM ESTAÇÕES FLUVIOMÉTRICAS DA BACIA HIDROGRÁFICA DO RIO IJUÍ - RS
}

\section{RELATIONSHIP BETWEEN AT-A-STATION HYDRAULIC GEOMETRY AND FLOW DURATION CURVE AT GAUGE STATIONS OF IJUÍ RIVER BASIN - RS}

\author{
Marco Alésio Figueiredo Pereira \\ Instituto de Pesquisas Hidráulicas, Universidade Federal do Rio Grande do Sul. \\ Av. Bento Gonçalves, 9500, Campus do Vale, Porto Alegre/RS, Cep: 91501-970, Brasil. \\ E-mail:geocram@gmail.com
}

Masato Kobiyama Instituto de Pesquisas Hidráulicas, Universidade Federal do Rio Grande do Sul. Av. Bento Gonçalves, 9500, Campus do Vale, Porto Alegre, RS, Cep: 91501-970, Brasil. E-mail: masato.kobiyama@ufrgs.br

Nilza Maria dos Reis Castro

Instituto de Pesquisas Hidráulicas, Universidade Federal do Rio Grande do Sul. Av. Bento Gonçalves, 9500, Campus do Vale, Porto Alegre/RS, Cep: 91501-970, Brasil.

E-mail:Nilza@iph.ufrgs.br

\section{Informações sobre o Artigo}

Data de Recebimento: 06/03/2014

Data de Aprovação:

$30 / 06 / 2014$

\section{Palavras-chave:}

Geometria Hidráulica, Curva de Permanência, Rio Ijuí.

Keywords:

Hydraulic geometry, Flowduration curve, Ijuí river.

\section{Resumo:}

Neste trabalho foi determinada a geometria hidráulica da seção em oito estações fluviométricas, instaladas na bacia do rio Ijuí $\left(10.650 \mathrm{~km}^{2}\right)$, região noroeste do Estado do Rio Grande do Sul. Para isso foram utilizados dados de medição de vazão realizada no decorrer do tempo de monitoramento de cada estação fluviométrica, com a série histórica variando de 11 a 72 anos. Além disso, com estes dados foi determinada a curva de permanência de vazões para cada estação e estimados seus $\mathrm{Q}_{5}, \mathrm{Q}_{50}$ e $\mathrm{Q}_{95}(5 \%, 50 \%$ e $95 \%$ de tempo que uma vazão é igualada ou excedida). Sabendo-se os respectivos $\mathrm{Q}_{\mathrm{s}}$ de cada seção analisou-se o comportamento dos expoentes $b, f$ e $m$, expoentes estes referentes às variáveis $w$ (largura), $d$ (profundidade) e $v$ (velocidade), respectivamente. Os resultados mostram que a variável $v$ tem maior sensibilidade sobre as oscilações de $Q$, seguido por $d$ e por último $w$. Isto pode ser explicado pelas feições geomorfológicas presentes na bacia, rochas basálticas, resultando em canais encaixados com margens bem definidas. Com isso pode-se concluir que a informação da geometria hidráulica da seção pode auxiliar a quantificação dos recursos hídricos de uma determinada bacia. 


\begin{abstract}
:
The present study determined the at-a-site hydraulic geometry at eight river-gauge stations, installed in the Ijuí river basin $\left(10,650 \mathrm{~km}^{2}\right)$ northwestern Rio Grande do Sul State. Discharge data obtained at these stations during the period from 11 to 72 years were used to determine the at -a-site hydraulic geometry of each station. Besides, with these data, the flow duration curves to each station and its estimated $\mathrm{Q}_{5}, \mathrm{Q}_{50}$ and $\mathrm{Q}_{95}(5 \%, 50 \%$ and $95 \%$ of time where a discharge is equaled or exceeded) were determined. Knowing their $\mathrm{Q}_{\mathrm{s}}$, analyzed the behavior of the exponents $b, d$ and $m$, which are exponents on the variables $w$ (width), $d$ (depth) and $v$ (velocity) respectively. The results show that the variable $v$ has a greater sensitivity to change of $Q$, followed by $\mathrm{d}$ and finally $w$. This can be explained with the fact that the studies basin is controlled by the predominant lithology, i.e., basalt, and that the channel is embedded and stable. Thus it can be concluded that the information of at-a-site hydraulic geometry may help to quantify water resources in a basin.
\end{abstract}

\section{Introdução}

O conhecimento dos recursos hídricos é primordial para o desenvolvimento e manutenção da sociedade, pois tendo-se conhecimento sobre a água se pode quantificar, analisar, determinar, priorizar e outorgar os seus diversos usos. Entretanto, para se ter uma visão holística deste bem que é de domínio público, limitado, e dotado de valor econômico deve-se ter também o conhecimento sobre sua distribuição espaço-temporal, sua qualidade, sua forma de circulação na bacia e seu armazenamento. Nesse sentido, uma técnica bastante difundida para a determinação quantitativa dos recursos hídricos em uma bacia hidrográfica, na escala temporal, pode ser a determinação da curva de permanência.

A curva de permanência pode ser expressa pela relação entre a magnitude e a frequência de vazões (Smakhtin, 2001). É apropriada para estabelecer rentabilidade econômica para empreendimentos hidroenergéticos, navegação em rios, abastecimento urbano e industrial, definir vazões ecológica, definir sistemas de irrigação, entre outros (Wolman \& Miller, 1960). Além disso, a curva de permanência é utilizada como critério de outorga em muitos estados brasileiros, por exemplo, Rondônia, Goiás, Alagoas e Paraná outorgam até 30\% da $\mathrm{Q}_{95}, 50 \%$ da $\mathrm{Q}_{95}, 90 \%$ da $\mathrm{Q}_{90}, 50 \%$ da $\mathrm{Q}_{95}$ de seus rios, respectivamente, onde $\mathrm{Q}_{50}, \mathrm{Q}_{90}$ e $\mathrm{Q}_{95}$ se referem a $50 \%, 90 \%$ e $95 \%$ de tempo que a respectiva vazão $(\mathrm{Q})$ é igualada ou excedida.

No momento que uma gota d'água passa do movimento laminar para o concentrado em canais, esta gota passa a ser integrante e agente modificador de uma rede de drenagem e das características fluviais de uma bacia hidrográfica. Christofoletti (1981) comenta que o fluxo d'água é o principal agente modificador de um canal de um rio. Suguio \& Bigarella (1990) abordam que as características fluviais são importantes não somente no que diz respeito aos recursos hídricos, tanto do ponto de vista da hidráulica e do controle da erosão, como também do ponto de vista geomorfológico, sedimentológico, e do planejamento regional. Salienta-se que o canal fluvial é integrante de uma rede de drenagem que por sua vez é produto das interações espaço-temporais dos regimes climáticos, hidrológicos, geológicos, morfológicas, pedológicos, e antropogênicos.

Nesse sentido, fazendo-se um caminho inverso a geometria hidráulica, proposta por Leopold \& Maddock (1953), através da determinação da forma do canal de uma seção transversal se apresenta com uma metodologia para se determinar a vazão ali passante, e por consequência a determinação da disponibilidade hídrica de uma bacia hidrográfica. Rhoads (1992) comenta que o conceito de geometria hidráulica de canais fluviais, constitui uma das primeiras aplicações da análise quantitativa em geomorfologia fluvial. Desde o anúncio desta teoria, foram realizados diversos trabalhos, sobre a geometria hidráulica na seção de medição e ao longo do canal. Estudos sobre esta teoria na forma de revisão bibliográfica se encontram em Singh (2003), Ferguson (1986), Grison \& Kobiyama (2011b).

Entretanto, no Brasil existem poucos trabalhos que abordam esta teoria, podendo-se citar os seguintes trabalhos: Christofoletti (1976) fez uma abordagem conceitual sobre este tema; Latrubesse \& Aquino (1998) analisaram a geometria hidráulica em rios da Amazônia Sul-Ocidental; Latrubesse \& Franzinelli (2002) analisaram as feições geomorfológicas constituintes do meio da planície Amazônica, com avaliação da geometria hidráulica na seção de Manacapuru no rio Solimões; Aquino et al. (2005) analisaram o comportamento hidrogeomorfológico do rio Araguaia, focalizando o regime hidrológico e as mudanças morfo-hidráulicas 
ocorridas no canal; Latrubesse (2008) avaliou padrões de ajuste de canal de grandes sistemas fluviais, empregando geometria hidráulica, vazão, largura, profundidade, inclinação, entre outros parâmetros em 10 rios da bacia Amazônica; Fernandez \& Bortoluzzi (2008) apresentaram resultados preliminares das relações da geometria hidráulica regional para rios das regiões Oeste e Sudoeste do Estado do Paraná; e Grison \& Kobiyama (2011a) analisaram a geometria hidráulica (da seção e a jusante) nas principais bacias hidrográficas paranaenses.

Analisando-se estes trabalhos observa-se que não houve estudos que abordaram a relação entre a geometria hidráulica e as curvas de permanência. Portanto, o objetivo do presente estudo é determinar os expoentes $b, f$ e $m$ da geometria hidráulica e correlacioná-los com a curva de permanência de cada estação fluviométrica. Como estudo de caso, o presente trabalho utilizou os dados das estações implantadas na bacia do rio Ijuí, Estado do Rio Grande do Sul.

\section{Conceitos}

\section{Geometria hidráulica}

A teoria da geometria hidráulica foi proposta por Leopold \& Maddock (1953) que a definiram como um modelo empírico com o intuito de analisar a largura, a profundidade, a velocidade e a carga sedimentar de um curso d'água natural, descrevendo a maneira pela qual as propriedades do canal fluvial mudam no decorrer do tempo e do espaço para suportar uma variedade de fluxos. Como a geometria hidráulica varia de um rio para outro e de uma seção para outra, então sua determinação é realizada na seção transversal e ao longo do canal fluvial (Figura 1).

Em determinada seção transversal a geometria hidráulica prevê as mudanças na largura, profundidade média e velocidade em relação às mudanças da vazão no decorrer do tempo, enquanto a geometria hidráulica em direção à jusante prevê a adaptação do tamanho e da forma do canal para uma vazão imposta, no decorrer do tempo e do espaço. Sendo que o canal busca um equilíbrio na sua forma, em virtude das forças atuantes, Lane (1937) comenta que uma série de fatores pode estabelecer e determinar a estabilidade e a forma do canal: (a) fatores hidráulicos (inclinação, rugosidade, profundidade, velocidade média, distribuição da velocidade, e temperatura); (b) forma do canal (largura, profundidade, e inclinações laterais); (c) natureza do

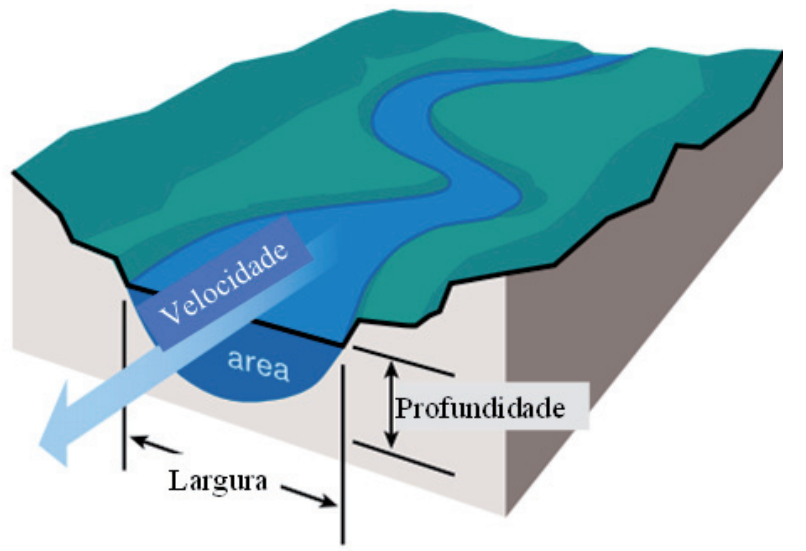

(a)

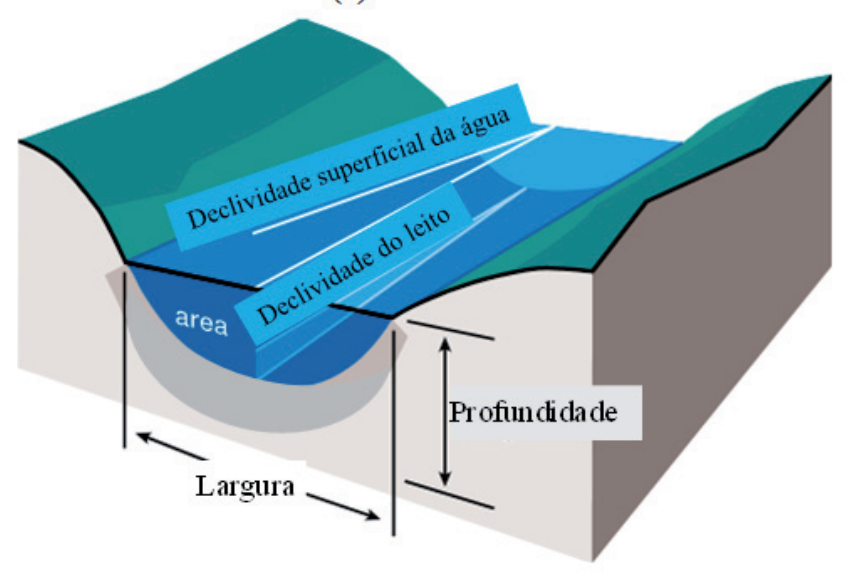

(b)

Figura 1 - Variáveis da geometria hidráulica. (a) em um perfil transversal; (b) em um perfil transversal e longitudinal. Fonte: adaptada de FISRWG, 1998).

material transportado (tamanho, forma, densidade, dispersão, quantidade, e material do leito e subleito); e (d) diversos (alinhamento, distribuição uniforme da vazão e envelhecimento do canal).

Analisando a geometria hidráulica no âmbito da seção transversal, Leopold \& Maddock (1953) estabeleceram as relações das variáveis da seção como funções potenciais diferenciadas somente pelos valores de seus expoentes e coeficientes, criando o termo geometria hidráulica, conforme as seguintes equações:

$$
\begin{aligned}
w & =a \cdot Q^{b} \\
d & =c \cdot Q^{f} \\
v & =k \cdot Q^{m}
\end{aligned}
$$

onde $Q$ é a vazão líquida $\left[\mathrm{m}^{3} / \mathrm{s}\right] ; w$ é a largura (nesse caso adotou-se a largura da linha d'água no momento 
da medição de vazão) $[\mathrm{m}]$; $d$ é a profundidade média (profundidade em relação a cota de medição da vazão) $[\mathrm{m}] ; v$ é a velocidade, determinada pela razão entre vazão líquida e área molhada da seção $[\mathrm{m} / \mathrm{s}] ; a, c$, e $k$, são coeficientes; e $b, f$, e $m$ são expoentes.

Em qualquer tempo e lugar estas variáveis estão inter-relacionadas pela equação de continuidade de massa.

$$
Q=w d v=(a c k) \cdot Q^{b+f+m}
$$

Portanto, obtêm-se que $b+f+m=1$ e $a c k=1$.

As modificações de $w, d$ e $v$ acontecem de acordo com as variações de $Q$, pois sob grande variedade de condições, essas variáveis hidráulicas aumentam como simples funções de potências positivas da vazão (LEOPOLD, 1994).

Vale ressaltar que as relações da geometria hidráulica são válidas somente para variações dentro da calha principal do canal do rio, no momento que há o extravasamento da calha estas equações não se aplicam. Na Tabela 1 são apresentados os valores dos expoentes de alguns trabalhos realizados no mundo.

Summerfield (1991) aborda que com o aumento da vazão as variáveis dependentes alteram-se em diferentes categorias, de acordo com diferentes canais dos rios, dependendo de um certo número de fatores controladores. Quando o canal está sobre materiais finos e coesivos, a profundidade aumenta proporcionalmente mais rápida do que a largura. Quando os materiais do canal são grosseiros e não coesivos, a largura aumenta rapidamente, em resposta ao aumento da vazão. As influências dos materiais dos canais explicam que canais em regiões semi-áridas, com o aumento da vazão, a largura aumenta mais rapidamente do que a profundidade, enquanto que, em ambientes úmidos essa relação é inversa, a profundidade aumenta mais do que a largura.

\section{Curva de Permanência}

Vogel \& Fennessey (1994) definem curva de permanência como a relação entre a magnitude e frequência de vazões diárias, semanais, mensais (ou algum outro intervalo de tempo) para uma bacia hidrográfica em particular, fornecendo uma estimativa da percentagem de tempo que uma dada vazão é igualada ou excedida num período histórico. Os mesmos autores dizem que
Tabela 1: Valores dos expoentes de trabalhos sobre geometria hidráulica.

\begin{tabular}{|c|c|c|c|}
\hline Fonte & $\boldsymbol{b}$ & $f$ & $m$ \\
\hline $\begin{array}{l}\text { Leopold \& Maddock } \\
\text { (1953) }\end{array}$ & 0,26 & 0,40 & 0,34 \\
\hline Wolman (1955) & 0,04 & 0,41 & 0,55 \\
\hline $\begin{array}{l}\text { Leopold \& Miller } \\
\text { (1956) }\end{array}$ & 0,26 & 0,33 & 0,32 \\
\hline $\begin{array}{l}\text { Leopold \& Langbein } \\
\text { (1962) }\end{array}$ & 0,23 & 0,42 & 0,36 \\
\hline Langbein (1964) & 0,23 & 0,42 & 0,35 \\
\hline \multirow{2}{*}{ Scott (1966) } & 0,35 & 0,42 & 0,55 \\
\hline & 0,24 & 0,56 & 0,20 \\
\hline \multirow{6}{*}{$\begin{array}{l}\text { Leopold \& Skibitzke } \\
\text { (1967) }\end{array}$} & 0,16 & 0,30 & 0,52 \\
\hline & 0,06 & 0,43 & 0,53 \\
\hline & 0,04 & 0,36 & 0,61 \\
\hline & 0,08 & 0,41 & 0,52 \\
\hline & 0,27 & 0,20 & 0,53 \\
\hline & 0,10 & 0,40 & 0,49 \\
\hline \multirow[t]{2}{*}{ Stall \& Yang (1970) } & 0,23 & 0,41 & 0,36 \\
\hline & 0,29 & 0,40 & 0,31 \\
\hline \multirow[t]{2}{*}{ Knighton (1972) } & 0,11 & 0,56 & 0,33 \\
\hline & 0,23 & 0,27 & 0,50 \\
\hline Richards (1973) & 0,16 & 0,43 & 0,42 \\
\hline \multirow{4}{*}{ Riley (1978) } & 0,42 & 0,41 & 0,16 \\
\hline & 0,35 & 0,48 & 0,17 \\
\hline & 0,35 & 0,52 & 0,13 \\
\hline & 0,38 & 0,46 & 0,16 \\
\hline \multirow{2}{*}{ Lane \& Foster (1980) } & 0,37 & 0,37 & 0,25 \\
\hline & 0,32 & 0,32 & 0,36 \\
\hline $\begin{array}{l}\text { Latrubesse \& Aquino } \\
\text { (1998) }\end{array}$ & 0,12 & 0,53 & 0,35 \\
\hline Jowett (1998) & 0,18 & 0,31 & 0,43 \\
\hline Kale \& Hire (2004) & 0,21 & 0,45 & 0,33 \\
\hline Stewardson (2005) & 0,11 & 0,28 & 0,52 \\
\hline Aquino et al. (2005) & 0,03 & 0,50 & 0,46 \\
\hline $\begin{array}{l}\text { Grison \& Kobiyama } \\
\text { (2011a) }\end{array}$ & 0,08 & 0,36 & 0,54 \\
\hline
\end{tabular}




\begin{tabular}{llll} 
Ran et al. (2012) & 0,15 & 0,42 & 0,43 \\
Minímo & 0,18 & 0,29 & 0,53 \\
Média & $\mathbf{0 , 0 3}$ & $\mathbf{0 , 2 0}$ & $\mathbf{0 , 1 3}$ \\
Máximo & $\mathbf{0 , 2 1}$ & $\mathbf{0 , 4 5}$ & $\mathbf{0 , 3 4}$ \\
\hline
\end{tabular}

o primeiro uso da curva de permanência foi atribuído a Clemens Herschel por volta de 1880. Desde então esta metodologia é bastante usada em diversos setores de recursos hídricos. A curva de permanência também é objeto de regionalização de vazões em bacias com e sem dados (Rianna et al., 2011; Li et al., 2010; Masih et al., 2010), além disso, pode ser utilizada para definir vazões ecológicas (Benetti et al., 2003), entre outras aplicações citadas na literatura. Smakhtin (2001) menciona que a curva de permanência é um dos métodos mais informativos para mostrar a completa variação da vazão de um rio. Castellarin et al. (2004) concluíram que dados de vazão obtidos por cinco anos são suficientes para obter estimativas consistentes de curva de permanência de longo período.

\section{Área de Estudo}

A bacia do rio Ijuí ( $\left.10.650 \mathrm{~km}^{2}\right)$, se localiza na região noroeste do Estado do Rio Grande do Sul, em uma posição central do derrame basáltico sul-americano, sobre o planalto médio gaúcho com altitudes entre 420 e 700 m (SEMA, 2000). É formada principalmente pelos rios Potiribu, Conceição e Ijuizinho, todos afluentes da margem esquerda do rio Ijuí. O rio Ijuí por sua vez é afluente do rio Uruguai sendo sua foz localizada no município de Pirapó - RS.

A Figura 2 apresenta a espacialização das estações fluviométricas na bacia, bem como sua rede de drenagem com os rios principais. Segundo Borges \& Bordas (1990) a bacia do rio Potiribu, que é uma sub-bacia do rio Ijuí, é representativa da área do planalto basáltico sul americano que abrange 1 milhão de $\mathrm{km}^{2}$, em termos de relevo, tipo de solo, regime de precipitação, e uso do solo que é essencialmente agrícola. O clima da região está classificado como mesotérmico brando superúmido sem seca (Cfa), pela classificação de Köppen (Beltrame, 2000). As precipitações da região apresentam homogeneidade temporal e espacial, sem a presença de uma sazonalidade definida (Pereira et al., 2013).

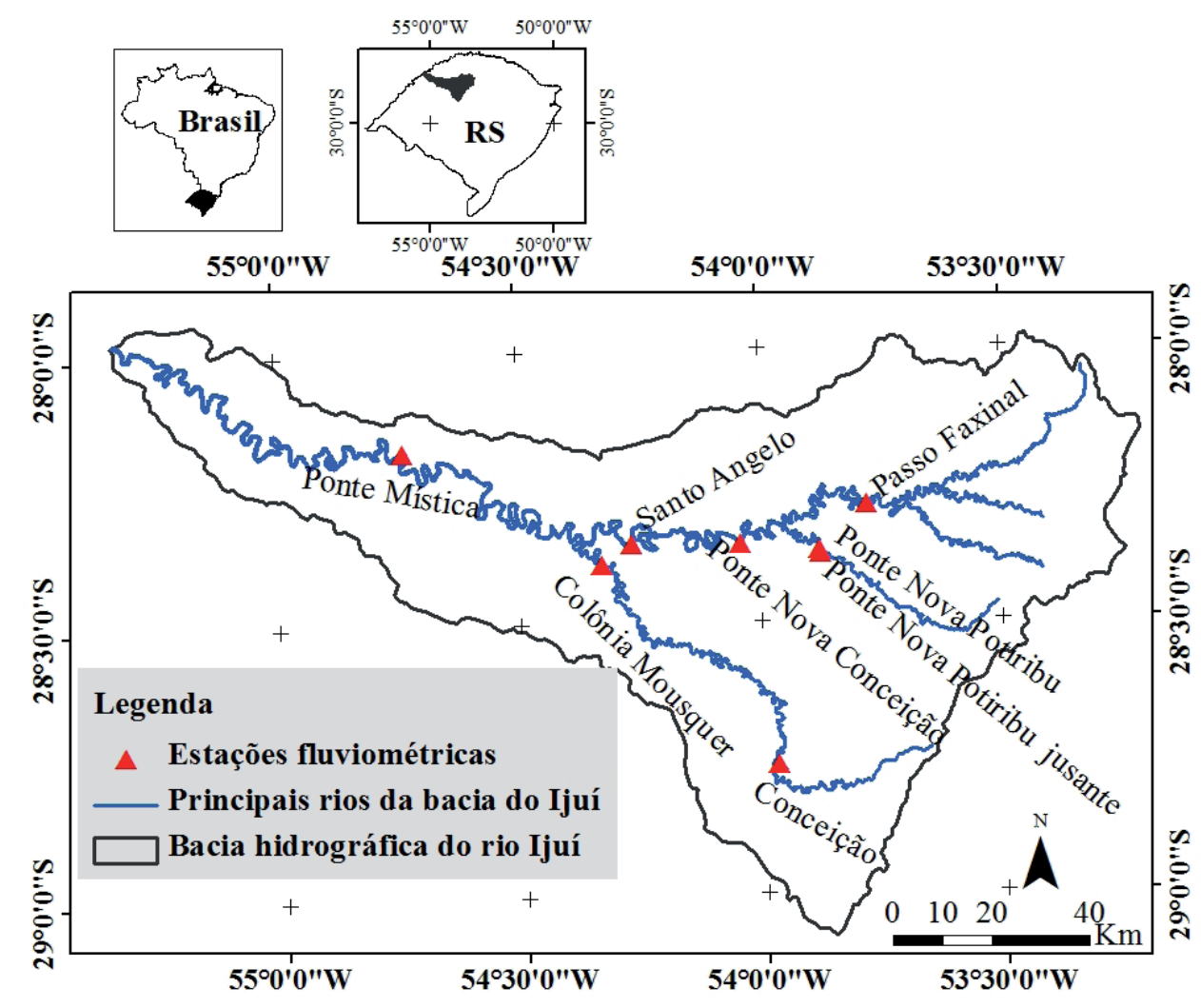

Figura 2 - Localização da bacia do rio Ijuí, com a espacialização das estações fluviométricas. 
O relevo da bacia é composto de coxilhas com declividades suaves entre 3 e $15 \%$, no qual, propicia o cultivo de grandes áreas e a mecanização agrícola. Esse planalto é situado sobre a rocha basáltica, formado por camadas oriundas da ocorrência de sucessivos derrames vulcânicos, datados de 120 milhões de anos. Entre essas camadas, depositaram-se camadas de arenito Botucatu que variam de alguns metros de profundidade até uma centena de metros (Leinz, 1949; IBGE, 1986).

\section{Materiais e Métodos}

\section{Dados utilizados}

No presente estudo foram analisadas informações hidrométricas e morfológicas do canal fluvial nas estações fluviométricas, implantadas na bacia do rio Ijuí, operadas pela (CPRM) Serviço Geológico do Brasil e sob responsabilidade da (ANA) Agência Nacional de Águas. Os dados foram obtidos do site da ANA no Sistema de Informações Hidrológicas (HidroWeb). Foi utilizado o software Hidro 1.2 como ferramenta de suporte para visualização e seleção dos dados, sendo selecionadas oito estações fluviométricas (Tabela 2).

As variáveis $(Q, w, d, v)$ foram obtidas no momento de cada medição da vazão, realizadas pela CPRM no decorrer do tempo de operação em cada estação, com a série histórica variando de 11 à 72 anos. Também faz parte monitoramento realizado pela CPRM, o levantamento do perfil transversal da seção de medição (levantamento altimétrico) que é realizado geralmente uma vez ao ano.

\section{Estabelecimento da Curva de Permanência}

Para definir as curvas de permanência, foram utilizados dados de $Q$ medidos em campo para cada estação. Estes dados foram ordenados em forma decrescente $\mathrm{e}$ determinado a sua frequência de excedência, calculada pela expressão $F=n / N$, sendo $F$ a frequência de excedência; $n$ a posição que o dado ocupa dentro da série histórica; e $N$ o tamanho da série histórica. Determinada a curva de permanência para cada estação estimou-se o $\mathrm{Q}_{5}, \mathrm{Q}_{50}$ e $\mathrm{Q}_{95}$ de cada estação, as quais se referem a 5\%, $50 \%$ e $95 \%$ de tempo que a respectiva vazão é igualada ou excedida.

\section{Análise da Geometria Hidráulica}

Os dados de cada medição $(Q, w, d$ e $v)$ foram dispostos em ordem decrescente, sendo $Q$ a variável determinante. Com estes dados agrupados e conhecendo

Tabela 2: Dados básicos das estações fluviométricas.

\begin{tabular}{|c|c|c|c|c|c|c|c|c|c|c|}
\hline Código & Nome & Rio & Município & Latitude & Longitude & $\begin{array}{l}\text { Área } \\
\left(\mathrm{km}^{2}\right)\end{array}$ & $\begin{array}{l}\text { Altitude } \\
\text { (m) }\end{array}$ & $\begin{array}{c}\text { Respon } \\
\text { sável }\end{array}$ & $\begin{array}{c}\text { Número } \\
\text { de } \\
\text { medições } \\
\text { de vazões }\end{array}$ & $\begin{array}{c}\text { Série } \\
\text { Histórica }\end{array}$ \\
\hline 75155000 & Passo Faxinal & Rio Ijuí & Ijuí & $28^{\circ} 17^{\prime} 21^{\prime \prime}$ & $53^{\circ} 46^{\prime} 48^{\prime \prime}$ & 1940 & 200 & ANA & 292 & $\begin{array}{c}\text { 25/11/1941 - } \\
07 / 09 / 2013\end{array}$ \\
\hline 75185000 & $\begin{array}{l}\text { Ponte Nova } \\
\text { Potiribu }\end{array}$ & Rio Ijuí & Ijuí & $28^{\circ} 22^{\prime} 38^{\prime \prime}$ & $53^{\circ} 52^{\prime} 32^{\prime \prime}$ & 609 & 320 & ANA & 169 & $\begin{array}{c}04 / 09 / 1963- \\
03 / 11 / 2001\end{array}$ \\
\hline 75186000 & $\begin{array}{c}\text { Ponte Nova } \\
\text { Potiribu - } \\
\text { Jusante }\end{array}$ & $\begin{array}{c}\text { Rio } \\
\text { Potiribu }\end{array}$ & Ijuí & $28^{\circ} 22^{\prime} 15^{\prime \prime}$ & $53^{\circ} 52^{\prime} 45^{\prime \prime}$ & 613 & 320 & ANA & 32 & $\begin{array}{c}03 / 06 / 2002- \\
05 / 09 / 2013\end{array}$ \\
\hline 75200000 & Conceição & $\begin{array}{c}\text { Rio } \\
\text { Conceição }\end{array}$ & Ijuí & $28^{\circ} 45^{\prime} 50^{\prime \prime}$ & $53^{\circ} 58^{\prime} 23^{\prime \prime}$ & 811 & 160 & ANA & 288 & $\begin{array}{c}12 / 06 / 1942- \\
04 / 09 / 2013\end{array}$ \\
\hline 75205000 & $\begin{array}{l}\text { Ponte Nova } \\
\text { Conceição }\end{array}$ & $\begin{array}{c}\text { Rio } \\
\text { Conceição }\end{array}$ & $\begin{array}{l}\text { Coronel } \\
\text { Barros }\end{array}$ & $28^{\circ} 23^{\prime} 01^{\prime \prime}$ & $54^{\circ} 01^{\prime} 54^{\prime \prime}$ & 970 & 160 & ANA & 114 & $\begin{array}{c}09 / 11 / 1974- \\
25 / 04 / 2013\end{array}$ \\
\hline 75230000 & Santo Angelo & Rio Ijuí & Entre Ijuis & $28^{\circ} 21^{\prime} 19^{\prime \prime}$ & $54^{\circ} 16^{\prime} 06^{\prime \prime}$ & 5440 & 200 & ANA & 285 & $\begin{array}{c}\text { 23/11/1941 - } \\
28 / 09 / 2013\end{array}$ \\
\hline 75295000 & $\begin{array}{l}\text { Colônia } \\
\text { Mousquer }\end{array}$ & $\begin{array}{c}\text { Rio } \\
\text { Ijuizinho }\end{array}$ & $\begin{array}{l}\text { Santo } \\
\text { Ângelo }\end{array}$ & $28^{\circ} 23^{\prime} 33^{\prime \prime}$ & $54^{\circ} 19^{\prime} 51^{\prime \prime}$ & 2160 & 200 & ANA & 118 & $\begin{array}{c}\text { 13/11/1974 - } \\
29 / 08 / 2013\end{array}$ \\
\hline 75320000 & Ponte Mística & Rio Ijuí & $\begin{array}{l}\text { São Luiz } \\
\text { Gonzaga }\end{array}$ & $28^{\circ} 10^{\prime} 53^{\prime \prime}$ & $54^{\circ} 44^{\prime} 18^{\prime \prime}$ & 9450 & 160 & ANA & 195 & $\begin{array}{c}22 / 10 / 1957- \\
14 / 08 / 2008\end{array}$ \\
\hline
\end{tabular}


os $\mathrm{Q}_{5}, \mathrm{Q}_{50}$ e $\mathrm{Q}_{95}$ de cada série histórica, foram determinados os expoentes $b, f$ e $m$ para as diferentes $Q$ de cada estação, conforme ilustra a Figura 3.

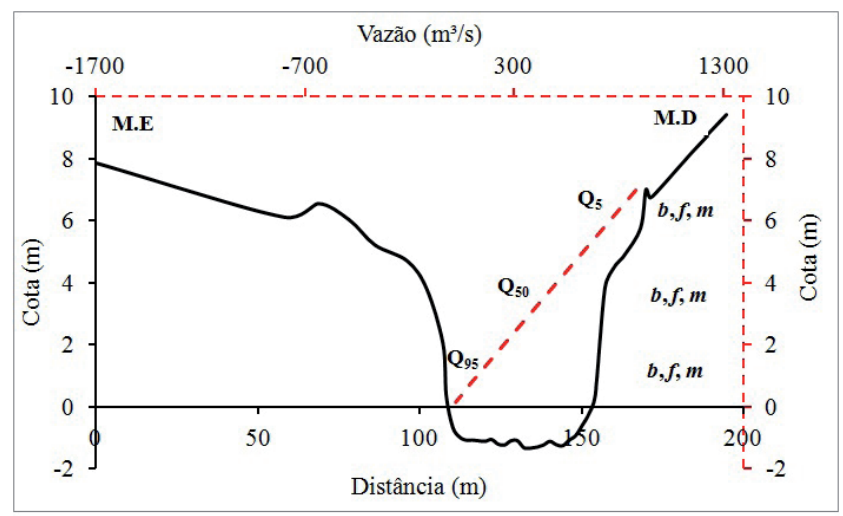

Figura 3 - Esquema ilustrativo do Q5, Q50 e Q95 de cada estação e seus respectivos expoentes $b, f e m$.

\section{Resultados e Discussões}

Sendo o objetivo deste trabalho determinar o comportamento dos expoentes $b, f$ e $m$ em relação a curva de permanência das vazões medidas em cada seção transversal, apresenta-se na Tabela 3 os diferentes valores dos respectivos expoentes em relação ao $\mathrm{Q}_{5}, \mathrm{Q}_{50}$ e $\mathrm{Q}_{95}$ de cada estação fluviométrica. Nota-se que o somatório $b+f+m$ foi igual a um em todas as $\mathrm{Q}_{\mathrm{s}}$ analisadas.

Comparando os valores apresentados na Tabela 3 com os valores apresentados na Tabela 1 observa-se que os valores encontrados pelo presente estudo apresentam certa discrepância dos dados apresentados pela literatura. Nos dados encontrados no presente trabalho o expoente $m$ apresentou valores significativamente superiores que aos outros dois expoentes $(b \mathrm{e}$ $f$ ), demonstrando que $v$ tem maior sensibilidade sobre as oscilações de $Q$. Isto pode ser explicado em virtude das feições geomorfológicas predominantes na bacia, conforme a descrição exposta por Leinz (1949) e IBGE (1986). Ou seja, em virtude da dureza das rochas predominante, basalto, os canais são encaixados com suas margens bem definidas e estáveis.

Leopold \& Maddock (1953) analisando a geometria hidráulica de 20 rios localizados nas partes central e sudoeste dos Estados Unidos chegaram a esta mesma constatação. Com o acréscimo da vazão, em média, a velocidade e a profundidade aumentam mais rapidamente do que a largura nas seções transversais.
A fim de entender pormenores a significância de cada expoente e sua relação com as $\mathrm{Q}_{\mathrm{s}}$ analisadas, a Tabela 4 apresenta o $\mathrm{R}^{2}$ de cada expoente. Pode-se constatar o alto valor dos $\mathrm{R}^{2}$, nos valores encontrados no expoente $m$ nos diferentes $\mathrm{Q}_{\mathrm{s}}$, seguido por $f$ e por último $b$. A equação de $\mathrm{R}^{2}$ é representada por $\sum_{i=1}^{n}\left(y_{i}-\bar{y}\right)^{2}$.

O expoente $m$ apresentou $\mathrm{R}^{2}$ médio de 0,$70 ; 0,75 \mathrm{e}$ 0,88 para $\mathrm{Q}_{5}, \mathrm{Q}_{50}$ e $\mathrm{Q}_{95}$, respectivamente. Já o expoente $f$ teve um $\mathrm{R}^{2}$ médio de 0,$61 ; 0,59$ e 0,65 para $\mathrm{Q}_{5}, \mathrm{Q}_{50} \mathrm{e}$ $\mathrm{Q}_{95}$, respectivamente, e por último o expoente $b$ exibiu valores médios de $\mathrm{R}^{2}$ em 0,64; 0,39 e 0,28 para $\mathrm{Q}_{5}, \mathrm{Q}_{50}$ e $\mathrm{Q}_{95}$, respectivamente.

\section{Relação $Q$ - w}

Os valores encontrados para o expoente $b$ foram os menores observados nas séries em análise. Nota-se que os referidos expoentes apresentam valores médios de 0,18 para $\mathrm{Q}_{5} ; 0,13$ para $\mathrm{Q}_{50}$ e 0,07 para $\mathrm{Q}_{95}$. Diante destas constatações, pode-se dizer que quando o fluxo d'água está com cotas altas, ou seja, em $\mathrm{Q}_{5}$ ocorre um pequeno aumento em $w$, provavelmente em virtude do formato do canal, como se exemplifica na Figura 3. Quando $Q$ está decrescendo ocorre uma diminuição na importância de $w$ sobre $Q$, provavelmente pelo mesmo motivo, a forma do canal (encaixado) não sofre alterações de $w$ em $\mathrm{Q}_{50} \mathrm{e}_{95}$.

Também, observa-se que na estação de Ponte Nova Potiribu, Ponte Nova Potiribu Jusante, Colônia Mousquer e Ponte Nova Conceição o expoente $b$ apresentou valores negativos em $\mathrm{Q}_{50}$ e $\mathrm{Q}_{95}$, o que jamais ocorreria na realidade. Isto implica uma baixíssima importância da variável $w$ nestas estações. Latrubesse \& Franzinelli (2002) observaram as mesmas constatações, e concluíram que no Rio Solimões ocorre grande estabilidade da largura e maior variação da profundidade e velocidade do rio com o aumento da vazão. Este comportamento da geometria hidráulica do Rio Solimões é típico de rios confinados ou de rios com margens estáveis e com material coesivo.

A Figura 4 apresenta os gráficos de dispersão $w$ vs. $Q$ para a estação de Passo Faxinal. Analisando a Figura 4 e conforme apresentado pela Tabela 4 fica evidenciado os baixos valores de $\mathrm{R}^{2}$ encontrados, esta constatação vai ao encontro dos baixos valores encontrados para o expoente $b$, que corrobora para a explicação que $w$ tem pouca importância sobre as oscilações de $Q$. 
Pereira M. A. F. et al.

Tabela 3: Valores dos expoentes $b, f$ e $m$ para estações fluviométricas

\begin{tabular}{|c|c|c|c|c|c|c|c|c|c|}
\hline \multirow{2}{*}{ Estações fluviométricas } & \multicolumn{3}{|c|}{ Q5 } & \multicolumn{3}{|c|}{ Q50 } & \multicolumn{3}{|c|}{ Q95 } \\
\hline & $b$ & $f$ & $m$ & $b$ & $f$ & $m$ & $b$ & $f$ & $m$ \\
\hline Passo Faxinal & $-0,93$ & 1,01 & 0,92 & 0,20 & 0,41 & 0,39 & 0,09 & 0,37 & 0,54 \\
\hline Ponte Nova Potiribu & 0,41 & 0,29 & 0,30 & 0,18 & 0,20 & 0,62 & 0,10 & 0,17 & 0,72 \\
\hline $\begin{array}{l}\text { Ponte Nova Potiribu } \\
\text { Jusante }\end{array}$ & 0,14 & 0,29 & 0,56 & 0,11 & 0,31 & 0,59 & 0,04 & 0,27 & 0,70 \\
\hline Conceição & 0,20 & 0,72 & 0,08 & 0,12 & 0,47 & 0,42 & 0,07 & 0,34 & 0,59 \\
\hline Ponte Nova Conceição & 0,12 & 0,31 & 0,58 & 0,02 & 0,27 & 0,70 & 0,02 & 0,22 & 0,77 \\
\hline Santo Angelo & 0,30 & 0,25 & 0,45 & 0,02 & 0,31 & 0,60 & 0,06 & 0,33 & 0,61 \\
\hline Colônia Mousquer & 0,22 & 0,33 & 0,45 & 0,13 & 0,38 & 0,49 & 0,08 & 0,36 & 0,56 \\
\hline Ponte Mistica & 1,00 & $-0,27$ & 0,27 & 0,24 & 0,20 & 0,56 & 0,12 & 0,23 & 0,64 \\
\hline Mínimo & $-0,93$ & $-0,27$ & 0,08 & 0,02 & 0,20 & 0,39 & 0,02 & 0,17 & 0,54 \\
\hline Média & 0,18 & 0,36 & 0,45 & 0,13 & 0,32 & 0,55 & 0,07 & 0,29 & 0,64 \\
\hline Máximo & 1,00 & 1,01 & 0,92 & 0,24 & 0,47 & 0,70 & 0,12 & 0,37 & 0,77 \\
\hline
\end{tabular}

Tabela 4: $\mathrm{R}^{2}$ dos expoentes $b, f$ e $m$ e sua relação $\operatorname{com} Q$.

\begin{tabular}{|c|c|c|c|c|c|c|c|c|c|}
\hline \multirow{2}{*}{ Estações fluviométricas } & \multicolumn{3}{|c|}{ Q5 } & \multicolumn{3}{|c|}{ Q50 } & \multicolumn{3}{|c|}{ Q95 } \\
\hline & $b$ & $f$ & $m$ & $b$ & $f$ & $m$ & $b$ & $f$ & $m$ \\
\hline Passo Faxinal & 0,27 & 0,24 & 0,91 & 0,49 & 0,74 & 0,56 & 0,38 & 0,91 & 0,89 \\
\hline Ponte Nova Potiribu & 0,83 & 0,67 & 0,73 & 0,43 & 0,40 & 0,87 & 0,37 & 0,40 & 0,92 \\
\hline $\begin{array}{l}\text { Ponte Nova Potiribu } \\
\text { Jusante }\end{array}$ & 1,00 & 1,00 & 1,00 & 0,27 & 0,89 & 0,87 & 0,09 & 0,89 & 0,97 \\
\hline Conceição & 0,31 & 0,78 & 0,04 & 0,23 & 0,61 & 0,58 & 0,19 & 0,61 & 0,83 \\
\hline Ponte Nova Conceição & 0,56 & 0,91 & 0,92 & 0,02 & 0,80 & 0,97 & 0,03 & 0,79 & 0,98 \\
\hline Santo Angelo & 0,74 & 0,42 & 0,64 & 0,56 & 0,51 & 0,76 & 0,41 & 0,67 & 0,87 \\
\hline Colônia Mousquer & 0,65 & 0,61 & 0,79 & 0,54 & 0,49 & 0,63 & 0,40 & 0,54 & 0,72 \\
\hline Ponte Mistica & 0,80 & 0,26 & 0,54 & 0,55 & 0,26 & 0,78 & 0,41 & 0,37 & 0,85 \\
\hline Mínimo & 0,27 & 0,24 & 0,04 & 0,02 & 0,26 & 0,56 & 0,03 & 0,37 & 0,72 \\
\hline Média & 0,64 & 0,61 & 0,70 & 0,39 & 0,59 & 0,75 & 0,28 & 0,65 & 0,88 \\
\hline Máximo & 1,00 & 1,00 & 1,00 & 0,56 & 0,89 & 0,97 & 0,41 & 0,91 & 0,98 \\
\hline
\end{tabular}

\section{Relação $Q-d$}

Os valores encontrados para o expoente $f$ apresentaram valores intermediários, com valores médios de 0,36 para $\mathrm{Q}_{5} ; 0,32$ para $\mathrm{Q}_{50}$ e 0,29 para $\mathrm{Q}_{95}$, apresentando certa semelhança. Os valores de cada expoente pode ser encontrado na Tabela 3. Diante destas constatações con- cluí-se a pouca influência da variável $d$ nestas estações. A Figura 5 apresenta os gráficos de dispersão $d$ vs. $Q$ para a estação de Conceição. Analisando o $\mathrm{R}^{2}$ da estação Conceição, bem como os $\mathrm{R}^{2}$ das outras estações para o mesmo expoente verifica-se uma maior significância de $d$ quando a vazão esta em $\mathrm{Q}_{5}$, isto se dá em virtude ao formato do canal (encaixado), ou seja, quando há o 
(a)

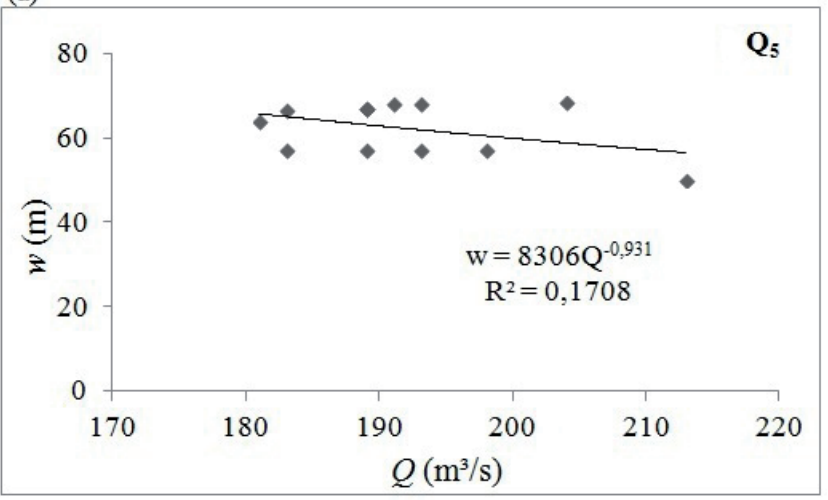

(b)

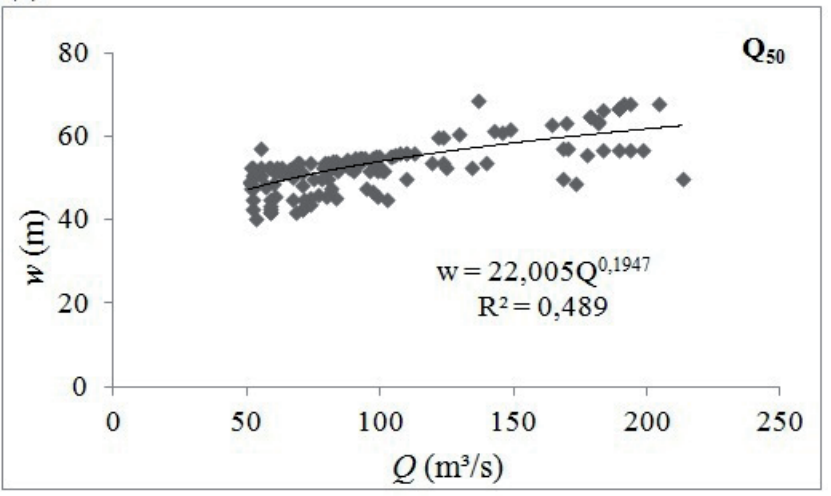

(c)

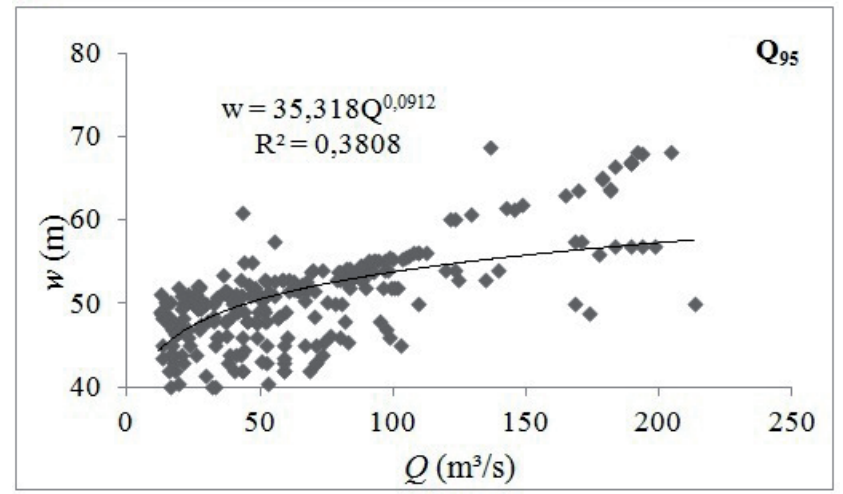

Figura 4 - Relação de w vs. $Q$ da estação Passo Faxinal: (a) Q5, (b) $Q 50$ e (c) $Q 95$.

aumento da vazão consequentemente há o aumento da profundidade. Quando as vazões estão em $\mathrm{Q}_{50}$ e $\mathrm{Q}_{95} d$ apresenta pouca diferença, ou seja, as oscilações de $Q$ não afetam significativamente $d$.

\section{Relação $Q$ - v}

Os valores para o expoente $m$ apresentaram as maiores médias, 0,45 para $\mathrm{Q}_{5} ; 0,55$ para $\mathrm{Q}_{50}$ e 0,64 para $\mathrm{Q}_{95}$. Percebe-se que com a diminuição da cota ocorre aumento nos valores do expoente, evidenciando

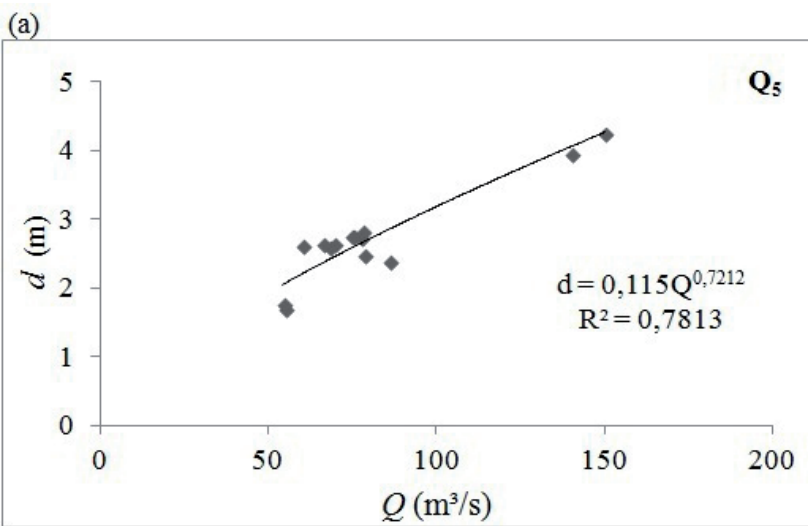

(b)

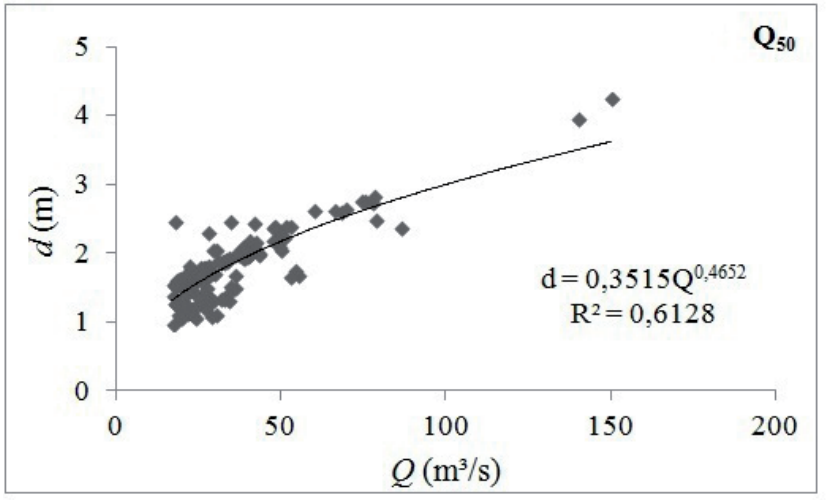

(c)

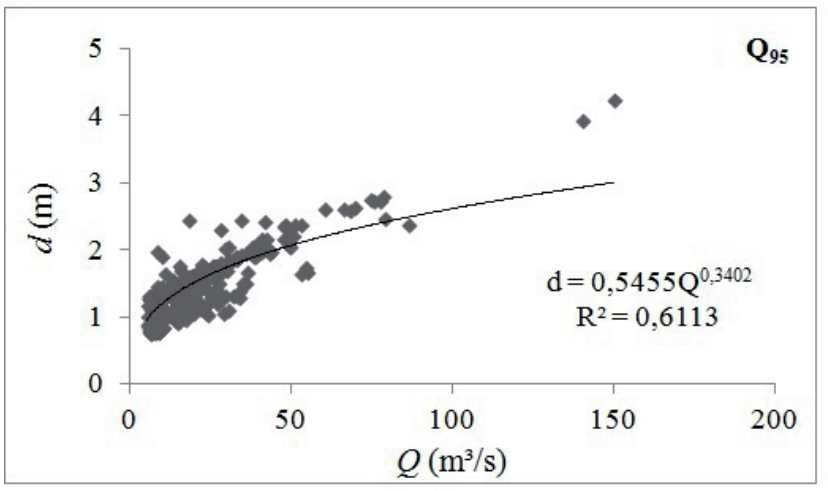

Figura 5 - Relação de d vs. $Q$ da estação Conceição: (a) Q5, (b) Q50 e (c) Q95.

a importância da variável $v$ sob os diferentes fluxos passante no canal, quanto mais baixa a cota maior a importância de $v$.

Isso pode ser respondido pela forma do canal pois os mesmos apresentam em sua maioria forma encaixada com margens fixas, constituída por materiais finos e coesivos, com maior poder de agregação e consequentemente menor probabilidade de alargamento de suas margens, e em muitos casos o leito do canal esta sob rocha sã (lajeado) o que proporciona maior velocidade 
do fluxo em virtude da menor rugosidade do canal.

A Figura 6 apresenta os gráficos de dispersão $v$ vs. $Q$ para a estação de Ponte Nova Potiribu. Os elevados valores do expoente $m$ apresentados na Tabela 3 e exemplificados na Figura 6 vão ao encontro dos valores de $\mathrm{R}^{2}$ apresentados na Tabela 4 comprovando a forte correlação entre $v$ e $Q$. Como dito anteriormente, os canais das respectivas estações fluviométricas apresentam $w$ bem definida, então com o aumento de $Q$ a variável $v$ aumenta significativamente (Figura 6), sendo que $v$ varia de 0,06 a $1,81 \mathrm{~m} / \mathrm{s}$.

(a)

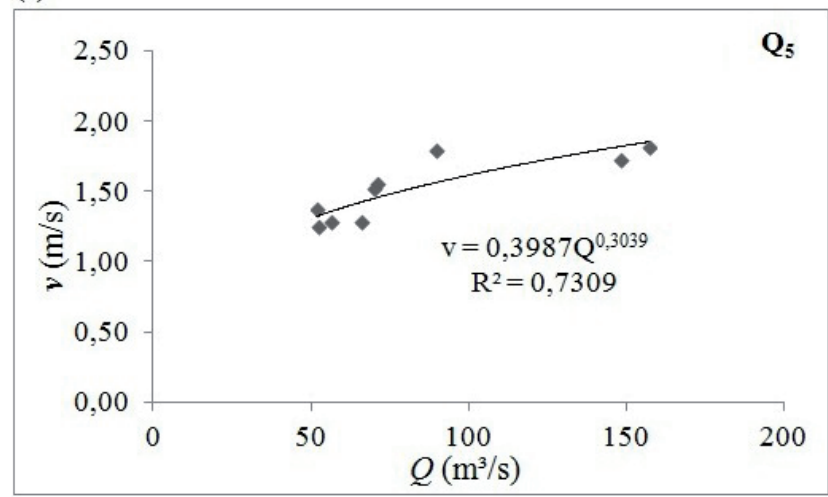

(b)

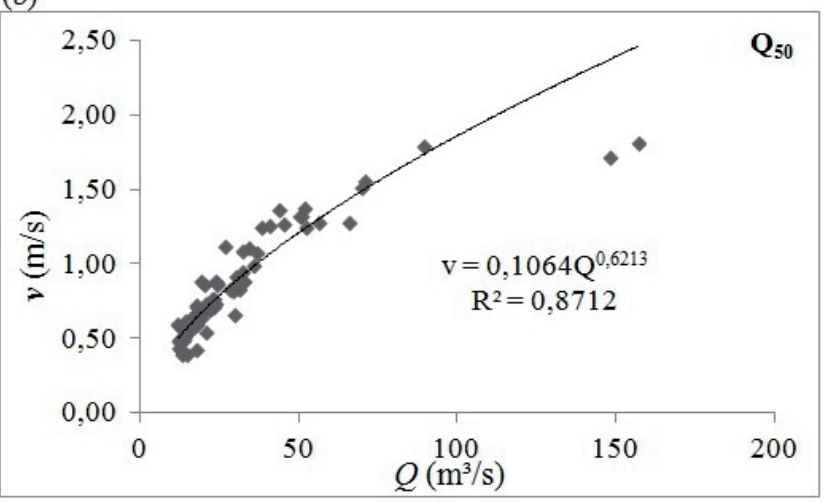

(c)

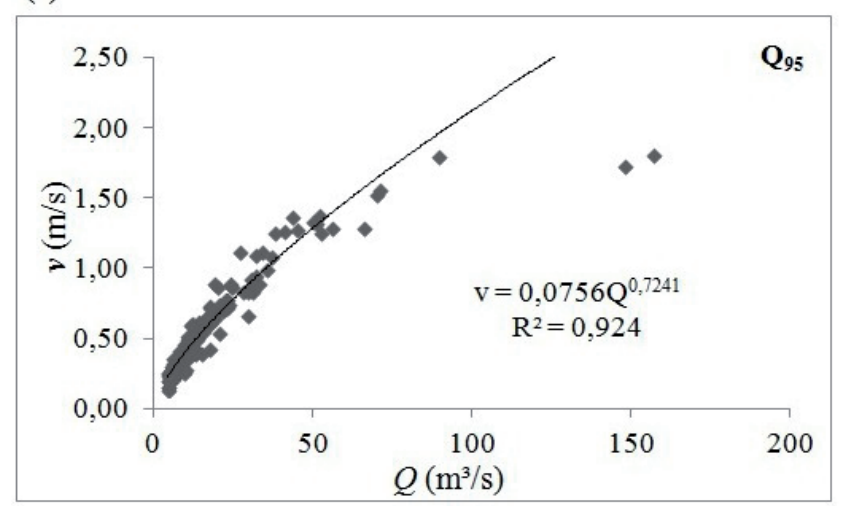

Figura 6 - Gráfico de dispersão v vs. $Q$ da estação Ponte Nova Potiribu: (a) Q5, (b) Q50 e (c) Q95.

\section{Conclusões}

O estudo da geometria hidráulica da seção transversal realizada em oito estações fluviométricas, localizadas na bacia do rio Ijuí, Estado do Rio Grande do Sul teve o objetivo de determinar os expoentes $b, m$ e $f$ da geometria hidráulica e correlacioná-los com a curva de permanência de cada estação fluviométrica analisada.

Avaliando os respectivos expoentes nas diferentes vazões $\left(\mathrm{Q}_{5}, \mathrm{Q}_{50}\right.$ e $\left.\mathrm{Q}_{95}\right)$ pode-se concluir que a variável $v$ tem maior correlação com $Q$. Isto pode ser explicado em virtude das estações fluviométricas estarem localizadas sob rochas basálticas, intercaladas com rochas sedimentares do arenito Botucatu. Consequentemente seus canais fluviais são encaixados e suas margens bem definidas e estáveis, deste modo, $w$ pouco se modifica em resposta ao aumento de $Q$, e à medida que $Q$ aumenta na seção transversal, $v$ aumenta rapidamente, seguido por $d$. $\mathrm{O}$ expoente $b$ apresenta valores próximos à zero em $\mathrm{Q}_{50}$ e $\mathrm{Q}_{95}$, isto se justifica pela homogeneidade litológica em toda a bacia do rio Ijuí. A variável $w$ aumenta de acordo com a área de influência de cada estação o que vai ao encontro da literatura existente que esclarece o aumento da seção do canal de acordo com o aumento da área a montante da seção de medição.

Para as diferentes $\mathrm{Q}_{\mathrm{s}}$ analisadas, os valores de $b, f$ e $m$ variaram de $-0,93$ a 1,00 ; de $-0,27$ a 1,01 e de 0,08 a 0,92 , respectivamente. As correlações de $Q-w$, $Q-d$ e $Q-v$ tiveram $\mathrm{R}^{2}$ médios de 0,$64 ; 0,61$ e 0,70 , respectivamente, para $\mathrm{Q}_{5}$. Para $\mathrm{Q}_{50}$ os valores de $\mathrm{R}^{2}$ médios para $Q-w, Q-d$ e $Q-v$ foram de 0,$39 ; 0,59$ e 0,75 , respectivamente, e para $\mathrm{Q}_{95}$ os valores foram de 0,28 ; 0,65 e 0,88 , respectivamente.

A geometria hidráulica através da determinação da forma do canal, através do conhecimento do comportamento de suas variáveis se apresenta com uma metodologia para se determinar a vazão ali passante, e por consequência a determinação da disponibilidade hídrica de uma bacia hidrográfica. Nesse sentido, pode-se predizer, se a forma da seção transversal de um determinado local e a forma do canal no decorrer do canal é o produto das interações dos mecanismos hidrológicos, geológicos, geomorfológicos e pedológicos ocorrentes na bacia, então se pode predizer que, sabendo-se a forma do canal, podemos inferir a vazão passante neste canal. 


\section{Referências Bibliográficas}

AQUINO, S.; STEVAUX, J. C.; LATRUBESSE, E. M.; Regime hidrológico e aspectos do comportamento morfohidráulico do Rio Araguaia. Revista Brasileira de Geomorfologia, n. 2, p. 29-41, 2005.

BELTRAME, L. F. de S. (coord.). Consistência de Dados Hidrológicos da Bacia Hidrográfica do Alto Uruguai, SubBacia 75. Porto Alegre, Instituto de Pesquisas Hidráulicas da UFRGS, 2000.

BENETTI, A. D.; LANNA, A. E.; COBAlCHINI, M. S.; Metodologias para determinação de vazões ecológicas em rios. Revista Brasileira de Recursos Hídricos v. 8 n. 2, p. 149-160, 2003.

BORGES, A. L.; BORDAS, M. P.; Escolha de bacias representativas e experimentais para estudo da erosão no planalto basáltico sulamericano In. Congresso Brasileiro e Encontro Nacional de Pesquisas sobre Conservação de solo, Londrina, Anais, 1990.

CASTELLARIN, A.; GALEATI, G.; BRANDIMARTE, L.; MONTANARI, A.; BRATH, A.; Regional flow-duration curves: Reliability for ungauged basins. Advances in Water Resources. v. 27, p. 953-965, 2004.

CHRISTOFOLETTI, A. Geometria hidráulica. Notícias Geomorfológicas, v. 16, n. 32, p. 3-37, 1976.

CHRISTOFOLETTI, A. Geomorfologia Fluvial. Editora Edgard Blucher, São Paulo, 313p. 1981.

FERNANDEZ, O.V.Q.; BORTOLUZZI, L.N.; Magnitude e freqüência das descargas dominantes em rios das regiões Oeste e Sudoeste do Paraná. Anais. VII Simpósio Nacional de Geomorfologia, Belo Horizonte (MG), UFMG, 11 p., CDRom., 2008.

FERGUSON, R. I., Hydraulics and hydraulic geometry. Progress in Physical Geography, v. 10, p. 1-31, 1986.

FISRWG. Stream Corridor Restoration: Principles, Processes, and Practices. Federal Interagency Stream Restoration Working Group. Item No. 0120-A; SuDocs No. A 57.6/2:EN 3/PT.653. ISBN-0- 934213-59-3. 1998.

GRISON, F.; KOBIYAMA, M.; Geometria hidráulica em bacias hidrográficas Paranaenses. Revista Brasileira de Recursos Hídricos, v. 16, n. 2, p. 111-131, 2011a.

GRISON, F.; KOBIYAMA, M.; Teoria e aplicação da geometria hidráulica: Revisão. Revista Brasileira de Geomorfologia, v. 12, n. 2, p. 25-38, 2011 b.
IBGE (Instituto Brasileiro de Geografia e Estatistica); Levantamento de recursos naturais, 33. Folhas $\mathrm{SH} 22$, Porto Alegre e parte das folhas: SH 21 Uruguaiana e SI 22 Lagoa Mirim. Projeto Radam Brasil, 792p. 1986.

JOWETT, I.G.; Hydraulic geometry of New Zealand rivers and its use as a preliminary methods of habitat assessment. River Research Applied v.14, p. 451-466, 1998.

KALE, V.S.; HIRE, P.S.; Effectiveness of monsoon floods on the Tapi River, India: role of channel geometry and hydrologic regime. Geomorphology, v. 57, p. 275-291, 2004.

KNIGHTON, A. D.; Changes in braided reach. Geological Society of America Bulletin, v. 83, p. 3813-3922, 1972.

LANE, E. W.; Stable channels in erodible material. Am soc. Civil Eng.,trans n.63 pp. 123-142 (1937)(Cap.III) In. River morphology. SCHUMM, S. A. (organizer) Colorado State University, 429p. 1972.

LANE, L. J.; FOSTER, G. R.; Modeling channel processes with changing land use. Proceedings, ASCE Symposium on Watershed Management, v. 1, p. 200-214, 1980.

LANGBEIN, W.B.; Geometry of river channels. Journal of the Hydraulics Division, ASCE, v. 90, n. HY2, p. 301-311, 1964.

LATRUBESSE, E. M.; AQUINO, S. Geometria hidráulica em rios da Amazônia Sul-Ocidental. Revista Geosul, Florianópolis, v. 14, n. 27 , p. $610-613,1998$.

LATRUBESSE, E. M; FRANZINELLI, E. The Holocene alluvial plain of the middle Amazon River, Brazil. Geomorphology, v. 44, p. 241-257, 2002.

LATRUBESSE, E. M. Patterns of anabranching channels: The ultimate end-member adjustment of mega rivers. Geomorphology, v. 101, p. 130-145, 2008.

LEINZ, V.; Contribuição à geologia dos derrames basálticos do sul do Brasil. Fac. Fil. Ciências e Letras da USP, Bol. CIII, Geologia 5, p. 1-61, 1949.

LEOPOLD, L.B.; MADDOCK, T.; The hydraulic geometry of stream channels and some physiographic implications. United States Geological Survey, Professional Paper, n.252, 56p. 1953.

LEOPOLD, L.B; MILLER J.P. Ephemeral streams-hydraulic factors and their relation to the drainage net. United States Geological Survey, Professional Paper, n.282A, 42p. 1956.

LEOPOLD, L. H.; LANGBEIN, W. B.; The concept of entropy in landscape evolution. United States Geological Survey, Professional Paper 500-A, 20 p. 1962. 
LEOPOLD, L. B.; SKIBITZKE, H. E., Observations on measured rivers. Geographical Annales, v. 49A, n. 2-4, p. 247-255, 1967.

LEOPOLD, L. B. A.; View of the River. Harvard University Press, Cambridge, Massachusetts, 290p. 1994.

LI, M.; SHAO, Q.; ZHANG, L.; CHIEW, F. H. S.; A new regionalization approach and its application to predict flow duration curve in ungauged basins. Journal of Hydrology, v.389, p. 137-145, 2010.

MASIH, I.; UHLENBROOK, S.; MASKEV, S.; AHMAD, M. D.; Regionalization of a conceptual rainfall-runoff model based on similarity of the flow duration curve: A case study from the semi-arid karkheh basin, Iran. Journal of hydrology v. 391, p. 188-201, 2010.

PEREIRA, M. A. F.; KOBIYAMA, M.; CASTRO, N. M. R.; Análise de homogeneidade da precipitação na bacia hidrográfica do rio Ijuí-RS. Anais XX Simpósio Brasileiro de Recursos Hídricos, Bento Gonçalves - RS, 2013.

RAN, L.; WANG, S.; LU, X. X.; Hydraulic geometry change of a large river: a case study of the upper Yellow River. Environmental Earth Science v. 66. p. 1247-1257, 2012.

RHOADS, B.L. Statistical models of fluvial systems. Geomorphology, v. 5, p. 433-455, 1992.

RIANNA, M.; RUSSO, F.; NAPOLITANO, F.; Stochastic index model for intermittent regimes: from preliminary analysis to regionalization. Natural Hazards Earth System Science. v. 11, p. 1189-1203, 2011.

RICHARDS, K. S. Hydraulic geometry and channel roughness-a nonlinear system. American Journal of Science, n. 273, p. 877-896, 1973.

RILEY, S. J.; The role of minimum variance theory in defining the regime characteristics of the lower Namoi-Gwydir basin. Water Resources Bulletin, v. 14, p. 1-11, 1978.
SCOTT, C. H.; Suspended sediment and the hydraulic geometry of channels. Erosion and Deposition in the Loess-Mantled Great Plains, Madison Creek Drainage Basin, Nebraska, United States Geological Survey, Professional paper 352-H, Washington, D. C., 1966.

SEMA - Secretaria Estadual de Meio Ambiente, Relatório Anual sobre a situação dos recursos hídricos do Estado do Rio Grande do e inventário hidrelétrico da sub-bacia 75. Tomo 1, v. 1, 526 p., 2000.

SINGH, V.P. On the theories of hydraulic geometry. International Journal of Sediment Research, v. 18, p. 196-218, 2003.

SMAKHTIN, V.U., Low Flow Hydrology: A Review. Journal of Hydrology v. 240, p. 147-186, 2001.

STALL, J. B.; YANG, C. T.; Hydraulic geometry of 12 selected stream systems of the United States. University of Illinois Water Resources Research Center, Research Report. n. 32, 1970.

STEWARDSON, M.; Hydraulic geometry of stream reaches. Journal of Hydrology v. 306, p. 97-111, 2005.

SUGUIO, K.; BIGARELLA, J. J.; Ambientes fluviais. $2^{\mathrm{a}}$ ed. Florianópolis: Ed. UFSC. 183 p. 1990.

SUMMERFIELD, M. A.; Global Geomorphology: An Introduction to the study of landforms. London: LONGMAN, 537 p. 1991.

VOGEL, R. M.; FENNESSEY, N. N.; Flow-duration curves. I. New interpretation and confidence intervals. Journal of water Resources Planning and Management. v. 120, n.4, p. 485504, 1994

WOLMAN, M. G.; The natural channel of Brandywine Creek, Pennsylvania. United States Geological Survey Professional Paper 271, Washington, D. C, 1955.

WOLMAN, M. G.; MILLER, J. P.; Magnitude and frequency of forces in geomorphic processes. Journal of Geology v. 68, p. 54-74, 1960. 